

\section{competencias desarrollan}

cuando utilizan

información y

comunicación?

RUTH MOLINA VÁSQUEZ

Coordinadora de la Especialización en Educación en Tecnología y Directora del Grupo de Investigación DIDACTEC de la Universidad Distrital Francis-

co José de Caldas

rmolinav@udistrital.edu.co

\section{Resumen}

Una de las inquietudes que se presentan de forma más reiterada entre los docentes, directivos y padres de familia, con respecto al uso de las Tecnologías de información y comunicación, es si esta inversión económica, en tiempo y esfuerzos se ven efectivamente reflejadas en los aprendizajes de los estudiantes. Esta también es una inquietud de las instancias encargadas de regir los destinos educativos del país. Tratando de buscar algunas respuestas en este sentido, se plantea una propuesta investigativa, en el marco del proyecto Meta Digital, que busca sistematizar los aprendizajes obtenidos por parte de los estudiantes, en términos de las competencias laborales básicas tecnológicas que desarrollan los estudiantes de educación media, en una muestra de nueve instituciones que asumen la propuesta pedagógica del proyecto. En este artículo, se presenta una descripción general esta investigación y algunos de los resultados obtenidos.

Palabras Clave: Aulas móviles, competencias laborales básicas tecnológicas, aprendizaje

\section{Abstract}

One of the concerns that have arisen over among teachers, administrators and parents, regarding the use of information and communication technologies, is whether students learn effectively reflects the financial investment, time and effort invested in the use of ICT. It is also a concern of government agencies responsible for governing the country's educational destination. Looking for answers in this regard, we present a research proposal under the Meta Digital Project, which aims to systematize the learning of students, job-related basic technological skills they develop in middle school, in a sample of nine institutions that assume the pedagogical approach of the project. This article presents an overview of research and some of the results. 


\section{Introducción}

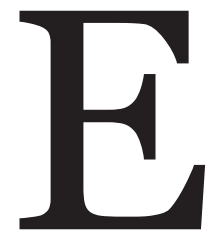

ste proceso de investigación se encuentra enmarcado en el área de educación del Proyecto Meta Digital, el cual es realizado por la Gobernación del departamento del Meta, el cual se ha venido desarrollando desde el año 2009, con la participación de en treinta instituciones educativas de veintinueve municipios, que incorporan aulas móviles como apoyo a los procesos de aprendizaje de los estudiantes de educación media.

Este proyecto que consiste en la aplicación de una propuesta pedagógica que se desarrolla en tres Fases: Sensibilidad, Acompañamiento y Sostenibilidad, mediante una estrategia basada en la formación, la asesoría y el seguimiento en aula, en torno a ejes de carácter didáctico, de diseño, tecnológico, todos ellos permeados por la estrategia de solución de problemas.

El contexto investigativo del proyecto se orienta en dos direcciones: la primera consiste en indagar acerca del tipo de apropiación que realiza la comunidad educativa, de las aulas digitales y de internet, a partir de su participación en el proyecto, y la segunda, en determinar el tipo de competencias que desarrollan los estudiantes y docentes de $10^{\circ}$ y $11^{\circ}$ que participan en el proyecto.

Dada la extensión del proceso y la diversidad de facetas del mismo, el presente artículo solo hace referencia a las competencias desarrolladas por los estudiantes, las cuales están enmarcadas dentro de las competencias laborales básicas tecnológicas, la cuales de acuerdo con un proceso de investigación anterior desarrollado en el departamento del Meta (Briceño, et all, 2008), no son expresas en los estándares de las áreas básicas de conocimiento, pero si están presentes en los aprendizajes manifestados por los estudiantes. De otra parte, las conclusiones presentadas, esta referidas a un proceso piloto realizado con nueve instituciones.

\section{Proceso metodológico utilizado}

La principal inquietud manifestada por la Gobernación del Departamento del Meta, en principio consiste en proporcionar a sus jóvenes, unas herramientas que les permitan desempeñarse laboralmente una vez terminen su educación media. Se considera que las tecnologías de información y comunicación pueden apoyar este propósito, pero surgen algunas dudas ¿Cuál estrategia utilizar para lograr este propósito?, ¿Qué se espera que "aprendan" los estudiantes? Y la más contundente de todas: si los estudiantes aprenden a partir de la apropiación de estas Tic, ¿Cómo evidenciarlo?

Se encuentra por una parte, que una estrategia coherente con el trabajo realizado por los estudiantes en este ciclo de formación es el Aprendizaje Basado en Problemas -ABP- y una manera de evidenciar estos aprendizajes consiste en caracterizar el tipo de competencias que desarrollan los estudiantes mediante el uso de la Tic, a partir de la implementación de la propuesta pedagógica del proyecto. Pero ¿Cuáles serán estas competencias? Luego de la revisión detallada de las competencias contenidas en los estándares de las áreas propuestas por el Ministerio de Educación Nacional, se llega a la conclusión que estas no incluyen las competencias tecnológicas, por lo cual se hace necesario caracterizar este tipo de competencias.

A partir de este lugar común, el proyecto se orienta a abordar el impacto que las aulas móviles tienen en la formación en la educación media que reciben los estudiantes que participan en la propuesta, en términos de la cualificación de sus competencias básicas laborales tecnológicas.

El problema de investigación se especifica en el caso de la indagación con los estudiantes, en la siguiente

\section{pregunta de investigación:}


¿Cuál es la incidencia en el desarrollo de competencias laborales básicas - específicas tecnológicas en los jóvenes, que se generan con la estrategia de Aprendizaje Basado en Problemas -ABP- apoyada en el uso de aulas móviles, tableros digitales e Internet en las instituciones que participan en el proyecto de meta digital?

En coherencia con esta pregunta orientadora del proceso investigativo, se plante como objetivo general, determinar las características de las competencias que los actores del proceso educativo desarrollan, a partir de la implementación de una propuesta pedagógica de Aprendizaje Basado en Problemas -ABP- apoyada en el uso de aulas móviles, en la educación media de las instituciones educativas que participan en el proyecto de Meta digital.

Los objetivos específicos son:

- Determinar los tipos y características de las competencias laborales básicas y laborales específicas de carácter tecnológico, desarrolladas por los estudiantes de décimo y undécimo grado de las instituciones que participan en el proyecto de Meta Digital.

- Determinar las características de las competencias didácticas en enseñanza de matemáticas, ciencias naturales y español, que desarrollan los profesores de las instituciones participantes en el proyecto de Meta Digital, a partir de la implementación de la propuesta pedagógica apoyada en el uso de aulas móviles.

- Determinar los indicadores específicos que dan cuenta de los niveles de desarrollo de las competencias alcanzadas tanto por los estudiantes como por los docentes participantes en el proyecto.

- Determinar los indicadores que dan cuenta de los aspectos que intervienen en el desarrollo de competencias didácticas de los profesores que participan en el proyecto.
- Verificar los indicadores que dan cuenta del desarrollo de las competencias laborales básicas de los estudiantes de décimo y undécimo grado que participan en el proyecto.

Metodológicamente se asume una investigación integral, consistente en la articulación de métodos cualitativos y cuantitativos, que permitan identificar y describir el tipo de competencias desarrolladas por los estudiantes, a partir de las bondades que representa cada uno de los dos enfoques y la manera como la información cuantitativa y cualitativa se complementan en pos de determinar los procesos que se presentan en las instituciones participantes y además, explicar lo que sucede en la práctica pedagógica (Bonilla, Rodríguez, 1995).

Por lo anterior la metodología se enmarca en un paradigma holístico que conjuga elementos de carácter: 1) empírico y teórico, 2) aplicado, pues es realizada con propósitos prácticos a partir del desarrollo de un nuevo programa, 3) busca trascender el nivel descriptivo y aportar elementos de carácter interpretativo del fenómeno de incorporación de las aulas móviles al aula de clase, 4) de campo, puesto que se realiza In Situ en las instituciones participantes.

De esta manera se asume un diseño metodológico que desde lo cuantitativo se enmarca en un Diseño de grupo control no equivalente (Cambell y Stanley, 1970), de corte cuasiexperimental, dado que se carece de control total de estímulos y variables a pesar de que se cuenta con un diseño de procedimientos de tipo experimental. Se cuenta con grupos experimentales conformados de forma natural, sin ningún proceso aleatorización (Cambell y Stanley, 1970), en los que se realiza un pretest y un postest.

Se considera como única variable independiente el proceso pedagógico de implementación de la estrategia ABP, apoyada en el uso de aulas móviles, mientras que se proponen dos variables dependientes, determinadas por el desarrollo de la competencias laborales básicas 
tecnológicas en el caso de los estudiantes y el desarrollo de competencias didácticas en el caso de los profesores.

Como hipótesis de trabajo se plantea que la implementación de la estrategia ABP apoyada en el uso de aulas móviles en las instituciones que participan en el proyecto de Meta Digital, incide en el desempeño de competencias laborales básicas tecnológicas de los estudiantes de décimo y undécimo grado y en el desempeño de competencias didácticas de los profesores. La hipótesis nula se orienta de determinar que no se encuentra incidencia de la implementación de la estrategia ABP apoyada en el uso de aulas móviles en las instituciones que participan en el proyecto de Meta Digitaly el desarrollo de competencias laborales básicas tecnológicas en los alumnos de décimo y undécimo grado, ni en las competencias didácticas de los profesores.

Desde lo cualitativo se adopta la etnografía desde la concepción antropológica y cultural que busca describir el estilo de vida de un grupo de personas (Martínez, 1997). Esta investigación tiene un carácter holísta de los fenómenos globales en diferentes contextos y emplea una variada gama de técnicas para obtener sus datos (Goetz y LeCompte, 1988).

Las categorías de análisis contempladas para evidenciar las competencias laborales básicas tecnológicas de los estudiantes, se orientan a dar cuenta de la Solución de problemas, el manejo tanto de herramientas informáticas como de la información, el manejo del enfoque de ciencia, tecnología y sociedad, de la naturaleza de la tecnología y de comunicación.

La recolección de información durante el proceso de investigación se realiza mediante dos estrategias, que se realizan de forma paralela: La recolección permanente de diarios de campo y la aplicación de encuestas tipo Licker, a manera de pretest y postest. La población objeto de estudio está conformado por todos los estudiantes de grado décimo y once de nueve municipios del departamento del Meta.
El proceso de investigación se desarrolla en las siguientes fases: Revisión inicial de elementos teóricos y prácticos que guíen el desarrollo de la propuesta de investigación; Recolección y procesamiento de información, que incluye la elaboración de instrumentos, aplicación de pruebas de validez y aplicación a los grupos de estudiantes; Análisis estadístico e interpretación de los instrumentos cuantitativos, cualitativos y análisis de las categorías de planteadas; Elaboración de Conclusiones e informe final.

\section{Algunos elementos conceptuales de apoyo}

Los contextos educativos han cambiado en los últimos tiempos, en la media en que se han evidenciado cambios en el sector económico, cultural y social, se han generalizado procesos de eliminación de las fronteras, la globalización, la apertura de mercados y se han vivido grandes avances en las tecnologías de la información y la comunicación. Esto ha incidido en el contexto de vida de las personas, en la base de conocimiento construido por la humanidad y en las concepciones de mundo, lo que implica que las condiciones y exigencias para "sobrevivir" han cambiado y las personas necesitan actitudes diferentes que les permitan adaptarse en un mundo en permanente cambio.

En este momento el contexto es el planeta, lo cual hace que los conocimientos en todas las áreas sean complejos y el problema universal radique en cómo percibir esta globalidad multidimensional, cómo articular y organizar los conocimientos, para lo que es necesario una reforma del pensamiento (Morin, 1999)

La educación se enfrenta a saberes reducidos, compartimentalizados, problemas cada vez más poli disciplinares, multidimensionales, transversales, globales, que requiere acercar a las nuevas generaciones a un conocimiento pertinente, acorde con esas nuevas concepciones de contexto, globalidad, multi dimensionalidad y complejidad. 
Ello requiere que la educación forme a los jóvenes para responder ante diferentes situaciones, resolver problemas y adaptarse a esas nuevas condiciones de la sociedad actual. Jóvenes competentes para enfrentarse a la incertidumbre, al cambio, para desenvolverse con soltura en un contexto eminentemente tecnológico.

De acuerdo con los Estándares Nacionales de Estados Unidos, en

Tecnologías de Información y Comunicación (Tic) para Estudiantes (ISTE, 2007), para aprender efectivamente y vivir productivamente en un mundo cada vez más digital. Los estudiantes deberían saber y ser capaces de: a) ser creativos e innovadores, en términos de construir conocimiento y desarrollan productos y procesos innovadores utilizando las Tic; b) ser comunicativos y trabajar de forma colaborativa utilizando medios y entornos digitales; c) investigar y manejarla información, utilizando herramientas digitales para obtener, evaluar y usar información; d) tener un pensamiento crítico, solucionar problemas y tomar decisiones, mediante el desarrollo de habilidades para planificar y conducir investigaciones, administrar proyectos, usando herramientas y recursos digitales apropiados; e) ser ciudadanos éticos a partir de la comprensión de los asuntos humanos, culturales y sociales relacionados con las Tic y practicar conductas legales y éticas; $y, f)$ poseer conceptos relacionados con las Tic, demostrando la comprensión de sistemas y el funcionamiento de las TIC .

En Europa, las competencias en el uso de las Tic son recogidas generalmente en los currículos, como en el caso de Inglaterra, en donde se establecen cuatro etapas dividas por edades (1:5-7años, 2:7-11años, 3:1114años, 4:14-16años). En estas etapas se encuentran unas categorías generales de competencias iguales para todas las etapas, entre las cuales están: Conocimiento, destrezas y comprensión y aptitud de estudio (Del Cerro y Llorente, 2005).

Por su parte, en Francia las competencias se dividen en dos niveles. En el primero se establecen cinco grandes competencias (citado por Del Cerro y Llorente, 2005): a) dominar las bases de la tecnología, b) adoptar una actitud de buen ciudadano frente al uso de la información, c) Producir, crear, modifcar y editar textos, d) documentarse multimedialmente, y e) comunicarse utilizando correo electrónico. En el segundo nivel, las competencias generales son: a) manejar y utilizar datos numéricos, b) producir y aprovechar documentos, c) Informarse y documentarse, d) organizar información, e) comunicarse.

En el caso colombiano, la competencia describe lo que una persona debe ser capaz de hace en el desempeño de una función productiva, lo cual se evidencia en criterios de desempeño, rango de aplicación, conocimientos, comprensiones esenciales y evidencias requeridas (SED, 2004).

El Ministerio de Educación Nacional define las competencias laborales de la siguiente manera:
"Las competencias laborales son el conjunto de conocimientos, habilidades y actitudes que aplicadas o demostradas en situaciones del ámbito productivo, tanto en un empleo como en una unidad para la generación de ingreso por cuenta propia, se traducen en resultados efectivos que contribuyen al logro de los objetivos de la organización o negocio.” (MEN, 2003:5)

De acuerdo con el Ministerio de Educación Nacional, las competencias laborales se dividen en generales o específicas (MEN, 2003). Las primeras son las requeridas para desempeñarse en cualquier entorno social y productivo, sin importar el sector económico, el nivel del cargo o el tipo de actividad, pues tienen el carácter de ser transferibles y genéricas4. Además, pueden ser desarrolladas desde la educación básica primaria y secundaria, y por su carácter, pueden coayudar en el proceso de formación de la educación superior y, una vez terminado este ciclo, a una vida profesional exitosa. 
A su vez, las competencias laborales específicas están orientadas a habilitar a un individuo para desarrollar funciones productivas propias de una ocupación o funciones comunes a un conjunto de ocupaciones. En el sector educativo, estas competencias han sido ofrecidas tradicionalmente por las instituciones de educación media técnica.

En este marco de ideas, las competencias tecnológicas que aportan el uso de aulas móviles en los contextos educativos, se configuran como competencias laborales específicas, busca responder a los requerimientos del sector productivo, actualizar a los estudiantes en el rápido avance tecnológico del mundo y generar especializaciones tempranas en los estudiantes, para asumir con propiedad su proyecto de vida y facilitar la movilidad entre distintas áreas de trabajo y sin limitar la polivalencia que actualmente es muy valorada por los empresarios y de gran utilidad para cumplir con los requerimientos de adaptación al mundo de hoy (MEN, 2003)

Con base en la reflexión sobre las diversas categorías de competencias propuestas, se considera que en la aplicación de la estrategia pedagógica basada en el Aprendizaje basado en Problemas -ABP-, del Proyecto Meta Digital a nivel de formación, asesoría y seguimiento en el aula, puede desarrollar algunas competencias básicas laborales de carácter tecnológico (MEN, 2007), las competencias tecnológicas de los estudiantes (Del Cerro y Llorente, 2005) y su apropiación (Cabello y Moyano, sf), que pueden considerarse para la educación media y entre las cuales se desatacan las siguientes categorías:

- Solución de problemas: la capacidad que desarrollan los estudiantes para identificar las situaciones problemas de su contexto, identificar sus causas, los aspectos que lo componen para así poder comprenderlo, la capacidad de formular de forma clara, sencilla y coherente problemas del contexto en relación con las áreas de conocimiento.
- Creacióneinnovación: capacidad para proponer diversas soluciones a los problemas planteados a partir de ideas novedosas o innovadoras, utilizando materiales y/o programas diversos o a partir de otras soluciones.

- Manejo operativo: involucra la competencia de uso de las herramientas informáticas, lo cual significa tener la habilidad para su uso a nivel técnico, desarrollar criterios que les permitan a los estudiantes seleccionar la herramienta adecuada para cada trabajo de acuerdo con sus necesidades, su manejo fluido, y por supuesto, el manejo adecuado tanto de herramientas de comunicación como de herramientas de colaboración.

- Manejo de información: capacidad de los estudiantes para buscar, identificar y seleccionar información en la red, para lo cual es necesario que identifiquen de forma clara las fuentes de información de acuerdo con su ubicación, temáticas que tratan, características de la información que contienen, y con una mirada crítica de acuerdo con su coherencia y confiabilidad.

- Ciencia, Tecnología y sociedad: capacidad para identificar críticamente, no solo el concepto de tecnología, sino las relaciones que esta establece tanto con la ciencia como con los contextos sociales y culturales de la humanidad.

- Naturaleza de la tecnología: capacidad para identificar el concepto de tecnología, sus características y reconocer a las Tic como expresiones particulares y diferenciarlas de otras tecnologías.

- Comunicación: capacidad para establecer conexiones comunicativas con otras personas utilizando las Tic como mediadoras, reconocer las reglas de comunicación en la red y ponerlas en práctica en sus comunicaciones cotidianas, 


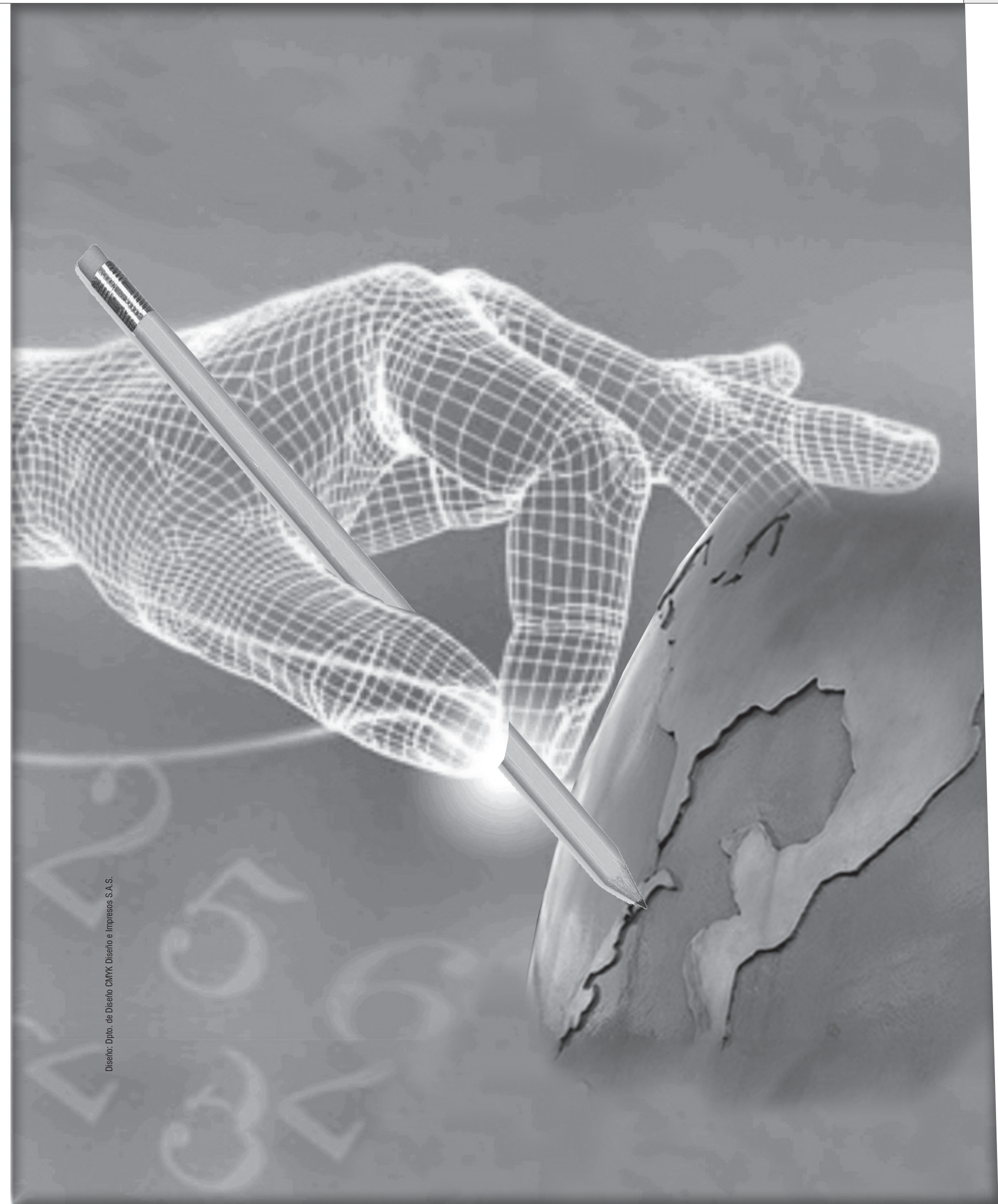


recocer las ventajas que ofrecen las Tic y hacer uso efectivo de las herramientas y plataformas a su alcance.

\section{Sobre el proceso de recolección de información y sus resultados}

Dada los dos aspectos metodológicos considerados para la realización del proceso investigativo, se presenta el proceso de recolección de información cualitativa y cuantitativa, desde la aplicación de encuestas y el registro permanente en diarios de campo durante el proceso de acompañamiento.

Esta información es recolectada en diez instituciones de los nueve municipios participantes en la Fase II del Proyecto, a partir de una muestra representativa de encuestas aplicada al $47 \%$ de total de la población de los estudiantes de $10^{\circ}$ y $11^{\circ}$ y un número total de 162 diarios de campo, recogidos a lo largo de seis meses.

Las encuestas se desarrollan mediante un cuestionario con escala de elección forzosa y con reactivos del tipo acuerdo y desacuerdo, que permite una de cinco posibles respuestas: completamente en desacuerdo, desacuerdo, inseguro, de acuerdo y completamente de acuerdo. Este tipo de instrumento se contesta de forma anónima y no permite incluir respuestas abiertas, pues incluye afirmaciones referidas a cada una de las categorías de análisis, según el caso, ante las cuales el encuestado selecciona una repuesta marcando en la respectiva columna.

Este instrumento se aplica antes de iniciar el proceso de implementación de la propuesta pedagógica (pretest) y nuevamente al finalizar el proceso (postest). Los análisis son codificados, tabulados, graficados y analizados de forma estadística.

Por su parte, las observaciones realizadas son de tipo no participante, a partir de las notas que se toman directamente en el aula de clase a medida que transcurren las actividades programadas previamente por el docente y el formador con apoyo de los computadores portátiles, internet y tablero digital . La observación se realiza en el periodo correspondiente a una hora de clase, el cual está entre 50 minutos y una hora y media y se realiza de forma descriptiva a partir de lo que sucede en la clase con los actores del proceso de enseñanza aprendizaje, los recursos utilizados, las estrategias planteadas, acciones realizadas y los comportamientos que tienen lugar. Estos diarios de campo son analizados con el apoyo un programa infromático.

Los resultados muestran en términos generales, que en seis de los diez casos, se registra en las instituciones de los municipios participantes, un mejor nivel en el manejo de las competencias básicas laborales tecnológicas de los estudiantes. Las categorías que presentan mejores niveles de logro son las de solución de problemas y creación e innovación, mientras que la categoría de comunicación es la que comparativamente, registra un menor nivel de ascenso.

Con respecto a la categoría de solución de problemas, los estudiantes perciben que han mejorado su capacidad de identificar problemas, dado que identifican sus causas y pueden dar argumentos que demuestran su comprensión de estos. Se muestran cambios positivos en la capacidad para formular problemas, puesto que pueden identificar los componentes del mismo y organizarlos de forma coherente. Esto se observa en la disminución de los porcentajes de estudiantes que se encuentran completamente en desacuerdo, en desacuerdo e inseguros frente a la presencia de estas competencias y el ligero aumento del porcentaje de estudiantes que se encuentran en acuerdo o completamente de acuerdo con poseer estas competencias.

Se observa en la implementación de la propuesta pedagógica, que en ocasiones, a pesar de que el diseño de la clase sea en relación con el ABP, persiste la transmisión de saberes, o una didáctica centrada en los 
contenidos, en la cual son evidentes los conocimientos del profesor sobre su disciplina, pero no las relaciones que establece con las posibilidades que ofrecen las TIC móviles incorporadas al proceso de enseñanzaaprendizaje, ni su conocimiento sobre las ideas de los estudiantes respecto al uso de aquéllas.

En otras ocasiones sucede lo contrario: se deja a los estudiantes que trabajen sin mayor orientación previa, como si se confiara demasiado en sus competencias para manejar información, seleccionar y utilizar herramientas de apoyo, y resolver problemas propuestos.

Los estudiantes a veces responden con una organización grupal espontánea con funciones bien distribuidas, lo que evidencia que trabajan con $\mathrm{ABP}$ y que ponen en juego sus competencias de solución de problemas y comunicación.

En algunos casos en donde la didáctica se inclina por la transmisión de contenidos o se centra en el estudiante, está ausente la socialización posterior sobre la solución de problemas o planteamiento de actividades, lo que daría al docente un conocimiento del contexto desde el cual se apoyan sus estudiantes para el trabajo en aula.

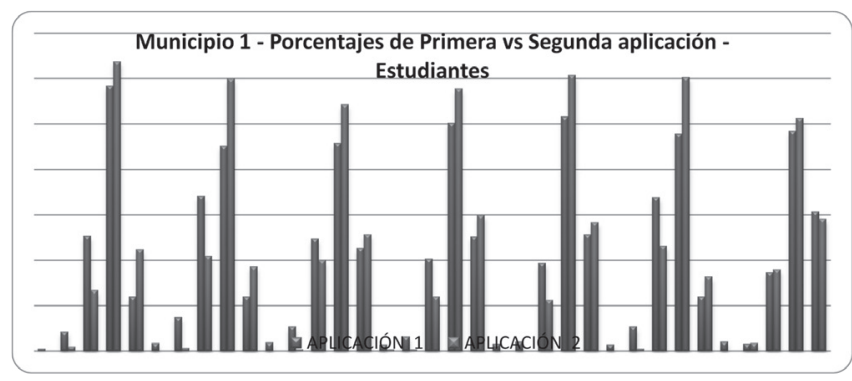

Grafico 2: Ejemplo de Resultados de las pruebas pretest y postest en uno de los municipios.

También se observan cambios en la categoría de Creación e innovación, que permiten afirmar que los estudiantes perciben un mayor nivel de desarrollo de sus competencias laborales básicas tecnológicas, referidas a la identificación y formulación de problemas, en donde se encuentra la identificación de sus causas, componentes y opciones de solución.

En la categoría de manejo operativo, se encontró un aumento significativo entre la primera y la segunda aplicación, en el porcentaje de estudiantes que se mostraron de acuerdo o completamente de acuerdo con poseer competencias relacionadas con el uso fluido de herramientas informáticas de propósito general, con el manejo de herramientas en red como correo electrónico, foros, chat, wikis y blogs.

La percepción de mejorar en las competencias referidas a esta categoría se corrobora con lo observado en la implementación de la propuesta pedagógica, pues los estudiantes demuestran en sus trabajos y actividades de clase, que manejan con cierta propiedad las herramientas básicas utilizadas para las clases.

Son pocos los casos en que los y las docentes, o los formadores, intervienen para aclarar a los estudiantes cómo utilizar una herramienta, un programa o una de las aplicaciones elegidas para las clases; las intervenciones de los docentes tienen que ver con correcciones de contenido:

“El docente encendió su computador portátil y llamó al primer grupo. Los estudiantes pasaron con una memoria USB en la que tenían la presentación... Una vez se abrió la presentación, los estudiantes se acercaron y el docente leyó cada una de las diapositivas, haciendo las correcciones que él consideraba pertinentes". Clase de Ciencias Naturales, grado 11

Aunque de manera incipiente, se advierte que la capacidad de apropiación de las expresiones tecnológicas, incide en la creatividad de los estudiantes para aprovechar mejor las herramientas y proporcionar comodidad en su uso.

Con respecto a la categoría de manejo de información, no se encuentran diferencias 
significativas en el desarrollo de competencias relacionadas con la capacidad para identificar, acceder y manejar efectivamente fuentes de información de acuerdo a su coherencia y utilidad, pues a pesar de que disminuye el porcentaje de estudiantes que se encuentran completamente en desacuerdo, los inseguros y los que están de acuerdo, aumentan el porcentaje de quienes están en desacuerdo y completamente de acuerdo.

Pese a lo anterior, durante la implementación se observa que es tal el interés de algunos estudiantes que en ocasiones usan una combinación de expresiones tecnológicas recientes (computador, internet, celular, tablero digital, etc.) con expresiones tecnológicas tradicionales (tablero, láminas didácticas, cuaderno, etc.) con el propósito de dar solución a un problema o ejercicio planteado.

Por supuesto tal práctica se empieza a observar de forma generalizada en las instituciones a media que pasa el tiempo de implementación y se logra advertir en algunas instituciones, que dicha combinación de expresiones tecnológicas se produce no pocas veces por iniciativa de los estudiantes, sin imposición o sugerencia de los profesores, lo cual sugiere que hay un manejo operativo y de información razonable en ambos casos.

De acuerdo con la percepción de los estudiantes, en la categoría de ciencia, tecnología y sociedad CTS- se presenta un leve aumento, en el desarrollo de las competencias relacionadas con la capacidad para identificar de forma crítica, las relaciones entre sociedad y tecnología, diferenciando el uso de la tecnología con propósitos sociales y la identificación de su impacto tanto en la sociedad como en la cultura.

Este leve cambio, se observa en el ligero aumento en los porcentajes de estudiantes que manifestaron estar completamente en completamente de acuerdo y de acuerdo, con poseer estas competencias y la disminución de los porcentajes en la opción en desacuerdo e inseguro.
En la categoría de naturaleza de la tecnología, la mayoría de los estudiantes se mostraron de acuerdo y completamente de acuerdo con poseer las competencias contenidas en esta categoría. El porcentaje de quienes estaban de acuerdo pasó o completamente de acuerdo, aumentan, mientras que diminuyen los porcentajes de completamente en desacuerdo, en desacuerdo e inseguro.

Esto indica que hay un aumento moderado en las competencias relacionadas con la identificación del concepto de tecnología, sus características, el nivel de explicación que se puede dar de ellas, y las diferencias que pueden establecer entre las TIC y otro tipo de tecnologías, tanto en sus componentes como avances logrados.

En la última categoría de comunicación, se presenta un aumento leve de los porcentajes de estudiantes que afirman estar de acuerdo y completamente de acuerdo, en contraste con la disminución del porcentaje de estudiantes que estuvieron completamente en desacuerdo e inseguros, entre la primera y la segunda aplicación, mientras que la opción de desacuerdo permaneció igual.

En la percepción de los estudiantes se observa polarización de opiniones, lo cual neutraliza el nivel de percepción de desarrollo de la competencia, referida a tener en cuenta las normas para comunicarse con otros utilizando las Tic, reconocer sus ventajas y hacer uso efectivo de ellas.

El análisis de los diarios de campo revela que a algunos docentes se les dificulta concebir propuestas de enseñanza a través de TIC. Por ello, aunque propongan problemáticas a resolver y propicien espacios de trabajo en equipo, la dinámica de algunos espacios de clase aún responde a la centralidad del docente en el proceso de aprendizaje.

Por su parte, algunos estudiantes tampoco logran vincular el uso de TIC con sus aprendizajes formales, adquiridos en el colegio. Muestra de ello es que el nivel 
de manejo operativo es bajo en herramientas poco novedosas por lo cual optan por volver a las tecnologías tradicionales.

Con respecto a la solución de problemas, se observa frecuentemente que mientras el docente se dirige a la clase para exponer el problema a resolver, algunos estudiantes lo ignoran, pues lo que les interesa es dar solución rápida a la actividad. Tratan de asumir la competencia como puente o pretexto para comunicarse con los pares más próximos:

"Mientras desarrollaban el ejercicio, los estudiantes se interrogaban entre ellos buscando colaboración para resolverlo, el docente hace preguntas sobre el taller pero pocos estudiantes atienden al docente ya que están más atentos de resolverlo directamente en su hoja con el apoyo de sus compañeros. A los diez minutos hace una socialización sobre las respuestas y da paso a desarrollar el segundo punto". Clase de Español, grado $10^{\circ}$

Estas situaciones muestran el rol activo asociado al activismo escolar: quien lo ejerce suele prescindir de la planeación, actúa de manera impulsiva, interesado únicamente en cumplir un propósito, una meta, sin que haya mayor claridad de la ruta que seguirá para alcanzarla (Briceño, et all, 2008).

Este tipo de desempeños sugieren la necesidad de seguir estimulando el trabajo en equipo desde pedagogías como el ABP e introducir nuevos elementos de reflexión en torno a las competencias laborales básicas tecnológicas. Pero al mismo tiempo insinúa que hay grupos de estudiantes entusiastas, activos, interesados en participar en esta clase de propuestas.

5. Algunas conclusiones transitorias

Es necesario aclarar que en el estudio inicialmente propuesto, se presentan en este artículo algunas conclusiones transitorias, dado que cubre los resultados de 10 instituciones ubicadas en nueve municipios del departamento del Meta de un total de 29 municipios. Los veinte municipios restantes, se encuentran en estos momentos en fases diferentes del proceso investigativo. Estas conclusiones se presentan entonces, a manera de reflexión sobre las características de las competencias evidenciadas, sobre los procesos educativos de las instituciones participantes, los procesos metodológicos de la investigación y algunas preguntas que surgen del proceso.

Inicialmente es necesario señalar que los resultados de este estudio piloto permiten evidenciar de forma efectiva, las siete categorías, en las que se agrupan las competencias propuestas, sin que durante el proceso se hubieran evidenciado otras categorías emergentes. Esto implica que se supera este primer nivel de validación del modelo de categorías y competencias propuestas.

Los resultados muestran que las competencias desarrolladas por los estudiantes en esta experiencia están ubicadas en múltiples aspectos, y los descriptores al interior de ellas también son variados. Esto implica que es necesaria su inclusión en los procesos de la educación media, dado que no aparecen en los Estándares para este nivel de formación y en las Orientaciones del área aparecen de forma parcial.

En aspectos específicos de las categorías de análisis, se encuentra que en dos de las instituciones hay algunos elementos que permiten evidenciar mejoras significativas en categorías como comunicación, manejo operativo y manejo de información. Paradójicamente, ninguna de ellas presenta mejora en la categoría de solución de problemas, mientras que en las restantes, es evidente. Tan solo una institución no se registra ningún proceso de logro en ninguna categoría, luego de la implementación de la propuesta pedagógica.

A partir del análisis general de esta información, se encuentra que el desarrollo del proceso de implementación de la propuesta pedagógica fue 
diferencial en las instituciones, debido a su organización interna, los tiempos dedicados, sus énfasis y las características particulares de su comunidad educativa. Así, se encuentra que la realización de procesos de formación con los estudiantes, no es garantía de niveles de logro en sus competencias básicas laborales tecnológicas. En cambio, si es de vital importancia la implementación realizada por los docentes en sus espacios de trabajo académico en el aula.

En algunos casos, los resultados de las encuestas son superiores a lo que se percibe en los registros de acompañamiento en aula a través del diario de campo. Asi, se evidencia un manejo básico de la estrategia $\mathrm{ABP}$ en los docentes y desorientación de los estudiantes, quienes no pueden formular efectivamente un problema, argumentar su comprensión o planear una solución apoyada en Tic. Esto lleva a plantear la necesidad de cualificar y seguir estimulando el trabajo en equipo desde el ABP e introducir nuevos elementos de reflexión en torno a las competencias laborales básicas tecnológicas.

Por el contrario, en otros casos se observa una percepción menor del logro de las competencias en relación con lo que se observa en el aula. Por ejemplo, en el caso de una institución, las encuestas muestran un nivel medio en las competencias de las categorías de manejo operativo y manejo de información, sin embargo se observa en el aula, un alto nivel por parte de los estudiantes, quienes sin la sugerencia de los docentes, incluyen el uso de otro tipo de tecnologías, integrándolas al desarrollo de las actividades, lo que resalta la importancia el conocimiento fáctico.

A pesar de que la estrategia de ABP, es compatible con enfoques pedagógicos de carácter constructivistaybusca desarrollar procesos de aprendizaje significativos, las actividades orientadas por los docentes y desarrolladas por lo estudiantes en clase, indican la presencia de dinámicas tradicionales a las que se integran el uso de las Tic y la estrategia de ABP. Esta puede ser una de las causas por la cual en la práctica se observa un manejo básico de esta estrategia, que indica que en el desarrollo de competencias se requiere de cambios que no son asunto exclusivo de recursos de apoyo como las Tic móviles; se requiere de un cambio de base en las formas de trabajo en el aula.

Llama la atención que en la categoría Comunicativa, que compendia las competencias orientadas a establecer conexiones comunicativas con otras personas utilizando las Tic como mediadoras, reconocer y hacer uso efectivo de estas herramientas, se observa en principio una percepción alta, que decrece en el postest. Sin embargo, en el trabajo de aula se evidencia un nivel de desempeño alto por parte de los estudiantes, lo cual puede evidenciar que se descubrieron otras herramientas y formas de comunicación desconocidas.

Finalmente, con respecto a la metodología empleada, se destaca que la combinación de elementos de carácter cualitativo y cuantitativo, enriquecen la mirada investigativa, permitiendo un mayor acercamiento al fenómeno estudiado. Sin embargo es necesario también destacar la enorme complejidad que ello implica en el análisis, dada la gran cantidad de información recolectada y los múltiples cruces a efectuar entre los elementos de carácter tanto cualitativo como cuantitativo.

NOTAS

El equipo de trabajo de este proyecto está conformado así: Mónica Rey-Gerente proyecto Meta Digital, Diana Rincón-Coordinadora, Sergio Briceño-Asesor, Ruth Molina-Asesora, Andrés Castellanos y Liliana Cadena-Auxiliares de investigación.

La traducción de este documento se encuentra en el portal de Eduteka, en: http://www. eduteka.org/pdfdir/EstandaresNETSEstudiantes2007.pdf

Se plantea inicialmente un formato de diario de campo indicado para realizar el registro de la observación, sin embargo en las actividades In Situ, este se realiza por parte de los formadores con un formato libre y puramente descriptivo.

\section{REFERENCIAS}

ALVAREZ, María Gladys (2007) Taller de investigación en investigación educativa: Los diarios de campo. En: http://www.javeriana.edu.co/ Facultades/Educacion/html/programa/maestria/ll-07/programas/ tall-2-ga.pdf. Consultado: Noviembre 12 de 2009.

BONILLA-CASTRO, Elsy., RODRÍGUEZ. Penélope. (1997) Más allá del dilema de los métodos. Editorial Norma. Santafé de Bogotá.

BRICEÑO, S., Molina, R., QUINTANA, A., RUIZ, A., CASTELLANOS, RUIZ, A.,VALBUENA W. (2008) Universidad Distrital Francisco José 
De Caldas. Grupo Didactec “Informe final”. Convenio Computadores Para Educar-Universidad Distrital-Municipio de Castilla la Nueva, Bogotá. Impreso

BRICEÑO, S., et all (2008) Computadores portátiles e Internet en la escuela. Universidad Distrital Francisco José de Caldas Computadores para Educar - Municipio de Castilla La Nueva. Documento de circulación interna.

BRIONES, Guillermo (1995) Métodos y técnicas de investigación para las ciencias sociales. Editorial Trillas, México.

CABELLO, Roxana., MOYANO, Renzo (2006) Competencias Tecnológicas y capacitación para la apropiación de las tecnologías. En: Razón y palabra. N. 49. Página 139.

CAMPBELL, D., STANLEY, J. (1970). Diseños experimentales y cuasiexperimentales en la investigación social. Amorrortu Editores. Argentina.

CASTELLANOS, 0., JIMENEZ, C., DOMINGUEZ, K (2009) Competencias tecnológicas: bases conceptuales para el desarrollo tecnológico en Colombia. En: Revista Ingeniería E Investigación Vol. 29 No. 1, Página 133-139.

DEL CERRO, Sonia., LLORENTE, María del Carmen (2005) DEL CERRO, Sonia., LLORENTE, María del Carmen (2005) Competencias tecnológicas en alumnos de secundaria y bachillerato. En: http:// tecnologiaedu.us.es/bibliovir/pdf/llorente.pdf Consultado: Agosto 15 de 2009.

ISTE (2007) Estándares nacionales de tecnologías de información y comunicación (tic) para estudiantes. NETS for Students: National Educational Technology Standards for Students, Second Edition. International Society for Technology in Education. En: http://www. iste.org Consultado: Abril de 2009

GOETZ, J.P., LECOMPTE, M.D. (1988) Etnografía y diseño cualitativo en investigación educativa. Ediciones Morata, Madrid.

KERLINGER, Fred (1988) Investigación del comportamiento. Editorial MacGrawHill. México.

LEON, O.G. y MONTERO, I. (2003). Métodos de investigación en Psicología y Educación ( $3^{\mathrm{a}}$ edición de Diseño de investigaciones). Madrid: McGraw-Hill.
MARTÍNEZ, Miguel (1997) La investigación cualitativa etnográfica en educación. Editorial Círculo de lectura alternativa. Bogotá.

MINISTERIO DE EDUCACION NACIONAL. (2003) Articulación de la educción con el mundo productivo: la formación de competencias laborales. En: http://www.mineducacion.gov. co/1621/articles-85777_archivo_pdf.pdf Consultado: Enero de 2009

MINISTERIO DE EDUCACION NACIONAL. (2003) Tendencias del mundo productivo y sus implicaciones en el perfil esperado en los trabajadores. En: http://www.mineducacion.gov.co/1621/ articles-85777_archivo_pdf1.pdf

MINISTERIO DE EDUCACION NACIONAL. (2007) Articulación de la educación con el mundo productivo: Competencias laborales generales

MINISTERIO DE EDUCACION NACIONAL. (2008) Diseño y ajuste de programas de formación para el trabajo bajo el enfoque de competencias. Bogotá.

MINISTERIO DE EDUCACION NACIONAL. (2008) Ser competente en tecnología: una necesidad para el desarrollo. MEN. Bogotá.

MOLINA, R., RUIZ, A. (2008) Más computadores, ¿más aprendizaje: Relación entre el uso de computadores 1 a 1 y el aprendizaje en la escuela. RibieCol. Barranquilla.

MORIN, Edgar (1999) Los siete saberes necesarios para la educación del futuro. Editorial Mesa Redonda Magisterio. Bogotá.

OSORIO, Carlos (2007) Enfoques sobre la tecnología. En: Revista Iberoamericana de Ciencia, Tecnología, Sociedad e Innovación. No.2 http://www.oei.es/revistactsi/numero2/osorio.htm Consultado: Enero 17 de 2009.

SECRETARÍA DE EDUCACIÓN DEL DEPARTAMENTO DEL META (2007), Plan de Desarrollo Educativo del Departamento del Meta 2008-2011. Villavicencio.

SECRETARÍA DE EDUCACIÓN DEL DEPARTAMENTO DEL META (2008). Diseño del Plan de apoyo al mejoramiento de la calidad de la educación - (2008 - 2011). Versión digital. 